\title{
Data-driven modelling captures dynamics of the circadian clock of Neurospora crassa \\ Amit Singh ${ }^{1,3,4}$, Congxin $\mathrm{Li}^{2}$, Axel C. R. Diernfellner ${ }^{1}$, Thomas Höfer ${ }^{2,5}$, and Michael Brunner ${ }^{1,5}$
}

1: Heidelberg University Biochemistry Center,

Im Neuenheimer Feld 328, 69120 Heidelberg, Germany

2: Theoretical Systems Biology (B086) Deutsches Krebsforschungszentrum, Im Neuenheimer Feld 267, 69120 Heidelberg, Germany

3: Institute for Biomechanics, ETH Zurich, Leopold-Ruzicka-Weg 4, 8093 Zurich, Switzerland

4: Tissue and Tumor Microenvironments Group, Kennedy Institute of

Rheumatology, University of Oxford, Oxford OX3 7FY, UK.

5: Corresponding authors.

michael.brunner@bzh.uni-heidelberg.de

t.hoefer@dkfz-heidelberg.de 


\section{Abstract}

Eukaryotic circadian clocks are based on self-sustaining, cell-autonomous oscillatory feedback loops that can synchronize with the environment via recurrent stimuli (zeitgebers) such as light. The components of biological clocks and their network interactions are becoming increasingly known, calling for a quantitative understanding of their role for clock function. However, the development of datadriven mathematical clock models has remained limited by the lack of sufficiently accurate data. Here we present a comprehensive model of the circadian clock of Neurospora crassa that describe free-running oscillations in constant darkness and entrainment in light-dark cycles. To parameterize the model, we measured high-resolution time courses of luciferase reporters of morning and evening specific clock genes in WT and a mutant strain. Fitting the model to such comprehensive data allowed estimating parameters governing circadian phase, period length and amplitude, and the response of genes to light cues. Our model suggests that functional maturation of the core clock protein Frequency (FRQ) causes a delay in negative feedback that is critical for generating circadian rhythms.

\section{Introduction}

Circadian clocks orchestrate daily cycles of biochemical, physiological and behavioral processes. Anticipation and adaptation to recurring environmental changes is thought to improve the fitness of organisms (Dunlap, 1999; Ouyang et al., 1998; Young and Kay, 2001). Circadian clocks share specific characteristics that are crucial for their function: (1) Circadian clocks generate in the absence of external stimuli a self-sustaining rhythm of about $24 \mathrm{~h}$. (2) They respond to recurring external stimuli such as changes in temperature, light, and nutrient sources to synchronize with the 24-hour environmental day-night cycle. (3) Circadian clocks are temperature compensated, such that the period length of circadian rhythms is over a broad range not significantly affected by the average 
daily temperature (Aschoff, 1965; Pittendrigh, 1954; 1960). Misalignment of the circadian clock and the environment contributes to several biochemical and physiological disorders including insomnia, mood disorder, diabetes, and cancer (Bass and Lazar, 2016; Bechtold et al., 2010; Challet, 2007; Hellweger, 2010).

Circadian clocks of eukaryotes are based on cellular transcriptional-translational feedback loops (TTFLs) regulating the expression of core clock genes as well as clock-controlled genes (Dunlap, 1999; Lee et al., 2000; Lowrey and Takahashi, 2000). In Neurospora crassa, the hetero-dimeric transcription activator White Collar Complex (WCC) and its inhibitor FFC, a complex containing Frequency (FRQ), FRQ-interacting RNA-helicase (FRH) (Cha et al., 2011; Guo et al., 2010) and casein kinase 1a (CK1a) (Gorl et al., 2001), are the core components of the TTFL (see below).

Previously, several mathematical models of the Neurospora circadian clock have been built on the basis of the core negative feedback loop constituted by the WCC and FRQ (FFC). Due to the unavailability of comprehensive experimental data these models uncovered and described principle properties of the clock in a rather generic manner and did, in most cases, not include detailed molecular interactions and mechanisms (Akman et al., 2008; Bellman et al., 2018; Dovzhenok et al., 2015; Francois, 2005; Gin et al., 2013; Hong et al., 2008; Leloup et al., 1999; Liu et al., 2019; Ruoff et al., 1996; Ruoff and Rensing, 1996; Tseng et al., 2012; Upadhyay et al., 2019, Upadhyay et al., 2020, Burt et al., 2021). In this study, we analyzed a WT and a mutant strain, $\Delta v v d$ (Malzahn et al., 2010), which is compromised in its capacity to adapt to light. We collected a comprehensive set of clock-related data by measuring in vivo in constant darkness and in light-dark cycles the expression of luciferase reporters of the core clock gene frq and of $v v d$. In addition, we measured luciferase reporters of conidial separation-1 (csp-1), which encodes a morning-specific clock-controlled repressor and one of CSP-1's target genes, fatty acid metabolim-3 (fam-3) (Sancar et al., 2011). The data warranted building a complex mathematical model with rather detailed molecular 
interactions. The data-driven model allowed us to estimate not only the expression phase but also the amplitude of rhythmically expressed genes and to uncover promoter-specific properties that determine their function in dark and light. Our approach demonstrates how high-resolution data sets can be used to more optimally exploit the theoretical capabilities of mathematical modeling.

\section{Results and Discussion}

\section{Interaction network of the Neurospora clock}

To understand how the manifold molecular interactions implicated in the circadian clock of Neurospora crassa control autonomous circadian oscillations and entrainment, we established an interaction network based on the available data (Figure 1): White Collar-1 (WC-1) and White Collar-2 (WC-2) are PAS (PERARNT-SIM) containing GATA-type zinc finger proteins, which constitute the heterodimeric White Collar Complex (WCC), the core transcription activator of the circadian clock of Neurospora (Crosthwaite et al., 1997; He et al., 2002; Linden and Macino, 1997; Ponting and Aravind, 1997; Talora et al., 1999). In the dark, transcription of wc-1 and wc-2 are controlled by unknown TFs (Kaldi et al., 2006). We modeled transcription of wcc being equivalent to the transcriptional production of its limiting component wc-1. The subsequent translation and assembly of the WC-1 and WC-2 subunits constituting the WCC was described by a single production term.

The WCC controls transcription of the core clock gene frequency (frq) (Aronson et al., 1994). FRQ protein was modeled as being initially inactive and unstable. $F R Q$ homo-dimerizes (Cheng et al., 2001a), assembles with FRH (Cheng et al., 2005), and CK1a (Gorl et al., 2001), forming the active FFC complex (Shi et al., 2010). The FFC and/or individual subunits or subcomplexes shuttle into the nucleus, receiving potentially licensing phosphorylation by CK1a (Gorl et al., 2001; Querfurth et al., 2007) and/or by other kinases (Diernfellner and Brunner, 2020; 
Huang et al., 2007; Mehra et al., 2009; Yang et al., 2003; Yang et al., 2002; Yang et al., 2001). As little molecular detail is known on assembly and maturation, we modeled these processes in a generic manner by a linear chain of six consecutive steps. The FFC is the core negative element of the TTFL. It interacts transiently with and inactivates the WCC by facilitating its phosphorylation by the CK1a subunit of the FFC (Schafmeier et al., 2005), and hence, we modeled the FFC acting enzymatically on the WCC and converting it into its phosphorylated, inactive form, P-WCC. As the WCC controls morning-specific transcription of frq, its inactivation closes the circadian negative feedback loop.

Inactivated phosphorylated WCC is stable and accumulates at elevated levels, and, hence, FRQ is part of a positive feedback loop with respect to WCC abundance (Cheng et al., 2001b; Crosthwaite et al., 1997; Denault et al., 2001; Dunlap, 1999; Lee et al., 2000; Neiss et al., 2008; Shi et al., 2010).

In the course of a circadian period $F R Q$ is progressively phosphorylated triggering its inactivation and degradation (Gorl et al., 2001; Larrondo et al., 2015). Inactivation of the FFC and degradation of its FRQ subunit was described by a single degradation/irreversible inactivation rate of the FFC. With decreasing amounts of active FFC, WCC is reactivated by dephosphorylation (Cha et al., 2008; Schafmeier et al., 2005) and replenished by de novo synthesis, and then a new circadian cycle begins with reactivation of frq transcription.

Our wiring schematic of the core circadian oscillator in the dark (Figure 1, upper left box) is topologically equivalent to the Goldbeter limit cycle oscillator model (Goldbeter, 1995). However, the Goldbeter model as well as other generic circadian oscillator models (Hong et al., 2008; Leloup et al., 1999; Ruoff et al., 1996; Ruoff and Rensing, 1996; Upadhyay et al., 2019) used a Hill-function for the transcriptional production of the core circadian inhibitors, PER in animals and FRQ in fungi, respectively, with Hill coefficients ranging from 2 to 7 (Bellman et al., 2018; Hong et al., 2008; Liu et al., 2019; Tseng et al., 2012). While the Hill-coefficients in these models helped generate robust oscillations, molecular interactions 
underlying such highly cooperative processes are not known. To attain smaller Hill exponents several previous models introduced a time delay by including intermediate steps in the negative feedback loop (Arcak and Sontag, 2006; Forger, 2011; Thron, 1991). A natural time delay is provided by the maturation of newly synthesized, inactive FRQ to active FFC, allowing us to describe WCC-activated transcription of frq by a simple Michaelis-Menten-like equation without introducing a Hill-coefficient.

The WCC controls rhythmic expression of many clock-controlled genes, among them vivid ( $v v d$ ) and conidial separation-1 (csp-1) (Froehlich et al., 2002; Malzahn et al., 2010; Sancar et al., 2015; Smith et al., 2010), which were included in our model.

Light cues directly activate the WCC and thereby reset the circadian clock and induce several cellular processes including biosynthesis of carotenoids, asexual conidiospores formation, and development of sexual structures (Corrochano, 2007; Harding and Turner, 1981; Lauter and Russo, 1991; Ruger-Herreros et al., 2014; Sancar et al., 2015; Sancar et al., 2011). The light-activated WCC is a potent transcription activator of frq and wc-1 (wcc in our model) as well as of many lightresponsive genes including $v v d$ and csp-1 (Chen et al., 2009; Froehlich et al., 2002; Malzahn et al., 2010; Sancar et al., 2015).

The WC-1 subunit of the WCC and VVD are blue-light photoreceptors harboring a flavin-binding light-oxygen-voltage- (LOV) domain (for review see (Demarsy and Fankhauser, 2009; Suetsugu and Wada, 2013)). VVD has no known function in the dark. Upon exposure to blue-light, a photo-adduct between a conserved cysteine residue of the LOV-domain and its bound FAD cofactor is formed (Zoltowski and Crane, 2008). WC-1 is activated in corresponding fashion (Cheng et al., 2003; Malzahn et al., 2010). The photo-adducts stabilize a conformation of the LOV domains that favors highly dynamic homo- and heterodimerization of VVD* and WCC* (Malzahn et al., 2010; Zoltowski and Crane, 2008). The lightactivated WCC homodimer (WCC* $\mathrm{WCC}^{\star}$ ) binds to light-response elements (LREs) 
(Froehlich et al., 2002). WCC*WCC* was modeled as a potent transcription activator of frq, vvd, wcc (wc-1), and csp-1 (Chen et al., 2009; Froehlich et al., 2002; Malzahn et al., 2010; Sancar et al., 2015; Smith et al., 2010). As the interaction of $\mathrm{VVD}^{*}$ with $\mathrm{WCC}^{*}$ leads to photoadaptation of light-dependent transcription, the activity of the $\mathrm{WCC}^{*} \mathrm{VVD}^{*}$ heterodimer and of monomeric lightactivated $\mathrm{WCC}^{*}$ was modeled as being equivalent to the activity of the dark form of the WCC. Light-activation triggers rapid hyperphosphorylation and accelerated degradation of WCC* (Kaldi et al., 2006; Linden and Macino, 1997; Schafmeier et al., 2008; Schafmeier and Diernfellner, 2011) and thereby also affects levels of its readily equilibrating complexes, $\mathrm{WCC}^{*} \mathrm{WCC}^{*}$ and $\mathrm{WCC}^{*} \mathrm{VVD}^{*}$. The light-activated, unphosphorylated $\mathrm{WCC}^{*}$ is unstable and rapidly degraded (Kaldi et al., 2006; Linden and Macino, 1997; Schafmeier et al., 2008; Schafmeier and Diernfellner,

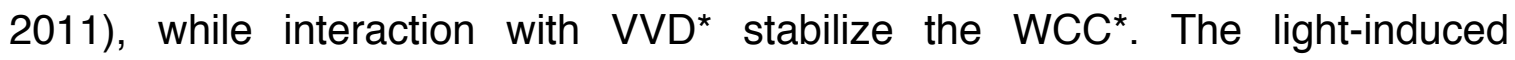
phosphorylation of $\mathrm{WCC}^{*}$ was not explicitly modeled but is included into the higher degradation rate of light activated species, which was constraint in our model to a half-time $\leq 3 \mathrm{~h}$.

In contrast, the FRQ dependent phosphorylation, inactivation and stabilization of all WCC species (Schafmeier et al., 2005) was explicitly modeled. The LOVdomain photo-adducts of WCC* and VVD* decay spontaneously into their dark forms with a half-time in the range of hours (Malzahn et al., 2010; Zoltowski and Crane, 2008).

All molecular reactions in the model were translated to a mathematical form describing either Michaelis-Menten or simple kinetics equations. The model contains 18 variables including mRNA and protein species. The detailed mathematical equations are displayed in Supplemental Information. The model includes the key features of the circadian clock and is sufficiently complex to allow an adequate, and mostly quantitative, molecular interpretation of experimental data.

High-amplitude response to light versus low-amplitude dark oscillations 
In order to collect sufficient data as a basis for the molecular model of the circadian clock we generated $W T$ and $\Delta v v d$ reporter strains expressing destabilized luciferase (lucPEST) (Cesbron et al., 2013) under the control of the morningspecific frq, $v v d$ and csp-1 promoters and the evening-specific fam-3 promoter. The lucPEST transcription units carried the 3' region of the trpC gene of Aspergillus nidulans for termination of transcription and the reporter genes were inserted downstream of the his-3 locus into the genome of Neuropora. Mycelial cultures of these strains were grown in 96-well plates (with more than 30 replicates in 3 independent experiments) on solid agar medium. The medium contained sorbose in order to restrict growth, which allows live recordings of bioluminescence over many days. The cultures were grown on the sorbose medium and synchronized by exposure to $12 \mathrm{~h}$ light, $12 \mathrm{~h}$ dark and $12 \mathrm{~h}$ light and then transferred to dark for $24 \mathrm{~h}$ before the luciferase measurement was started $(\mathrm{t}=0)$ (Figure 2). After 4 days in constant darkness $(\mathrm{t}=72 \mathrm{~h}$ ) the samples were exposed to $12 \mathrm{~h}$ light, $12 \mathrm{~h}$ dark and $12 \mathrm{~h}$ light, and then kept in the dark for another $64 \mathrm{~h}$. In constant darkness the expression levels of all lucPEST reporters oscillated but showed dampening over time (see Figure 2). It is not clear whether and to what extent the dampening is the consequence of desynchronization of individual nonconnected hyphae or due to a real reduction of amplitude of the oscillator.

The expression of $f r q, v v d$, and $c s p-1$ reporters oscillated with peaks levels in the subjective morning, while fam-3, which is repressed by CSP-1 in the morning, was rhythmically expressed in antiphase with peak levels in the subjective evening. As expected on the basis of previous studies (Cesbron et al., 2013; Elvin et al., 2005; Heintzen et al., 2001; Hunt et al., 2007), all oscillations were phase-delayed in $\Delta v v d$ (Figure 2, right panels). After the light was turned on, expression of frq was rapidly induced in $W T$ to a level $\sim 18$-fold higher, but then dropped and adapted to a level $\sim 12$-fold higher than in the dark. Expression of $v v d$ was rapidly induced $\sim 50$-fold compared with the level in the dark and then adapted to a $\sim 10$-fold higher level, and csp-1 was induced $\sim 6$-fold and fully adapted to its dark expression level. Thus responses of $f r q, v v d$, and $c s p-1$ to light had much larger amplitudes than 
autonomous oscillations in constant darkness. Moreover, the three light-inducible promoters responded with different inactivation kinetics to light-activated WCC.

In contrast, the evening-specific fam-3 reporter was transiently repressed after the light was turned on. This is consistent with the light-induced transient expression of CSP-1, which represses its own gene, csp-1, as well as fam-3 and many other genes (Sancar et al., 2011).

In $\Delta v v d$, the circadian oscillation of all reporters was phase-delayed (arrows in Figure 2) as expected. The transcriptional dynamics in light-dark cycles of frq and $v v d$ were quite different in $\Delta v v d$ as compared to $W T$. The light response of the $v v d$ reporter was qualitatively similar to that in WT. However, the spike level after light on was almost twice that of $W T$, and the light-adapted expression level was 5fold higher than in WT. These data are consistent with the increased activity of light-activated WCC in the absence of its light-dependent inhibitor, VVD (Malzahn et al., 2010). In contrast, the initial light-induced spikes of the frq reporter were similar in $\Delta v v d$ and $W T$, suggesting that the frq promoter was already functionally saturated at WT levels of light-activated WCC. After the initial light-induced spike, frq-IUCPEST expression levels decreased sharply and more markedly in $\Delta v v d$ than in $W T$, and then increased again in the further course of the light phase. When light was turned off, $v v d$ transcription decreased as expected, consistent with the decreasing level of light-activated WCC. Surprisingly, however, the frq level in $\Delta v v d$ increased transiently after the lights were turned off and then dropped. The difference in expression dynamics of frq compared with $v v d$ after light on and even more so after the light-to-dark transition is consistent with the previously reported refractory behavior of the frq promoter (Cesbron et al., 2015; Li et al., 2018). Indeed, in $\Delta v v d$ the frq promoter is partially repressed in light, and hence not maximally active. The rapid decrease of the light-induced transcription spike after light on reflects the dynamics of the light-dependent partial repression. Similarly, the transient increase in frq-IUCPEST transcription in $\triangle v v d$ after turning off the light is consistent with the repression of the frq promoter being relieved faster than the WCC activity (level) decreases. 


\section{The model quantitatively captures circadian oscillations and light entrainment in WT Neurospora}

Our model was trained to the temporal expression profiles of the frq, $v v d, c s p-1$ and fam-3 reporters in $W T$ and $\Delta v v d$. The parameter space was restricted to a biological meaningful range as described in Supplemental Information. The parameters were estimated using maximum likelihood (Isqnonlin optzimizer implemented in D2D/Matlab; (Raue et al., 2015; Sancar et al., 2011)).

Overall, our model simulations show oscillation of all reporters in the dark and responded in appropriate manner to the LD-cycles (Figure 3). The simulations of frq and $v v d$ transcription in WT captured the experimental data quite well, reproducing period length, phase and amplitude of the free-running oscillations as well as the transcription dynamics in LD cycles (Figure 3, left panels). The simulations of $\operatorname{csp}-1$ expression in WT captured period length and phase in DD and part of the dynamics in LD cycles, while amplitude of the model deviated somewhat from the measured data. In our model, we assumed that $\operatorname{csp}-1$ is rhythmically activated by the WCC and rhythmically repressed by CSP-1. The deviation of data and model could indicate that, in addition to WCC, an unknown transcription activator contributes to the $c s p-1$ expression. Furthermore, compared to the luciferase data, the model predicted slightly higher csp-1 levels towards the end of the $12 \mathrm{~h}$ light phases, and the level then dropped abruptly after lights-off in the model. This sudden drop in $\operatorname{csp}-1$ RNA, and hence of the short-lived CSP-1 repressor (Sancar et al., 2011), lead to a rapid relieve of fam-3 repression and thus, the model showed transiently slightly higher fam-3 levels than experimentally observed.

The simulations of gene expression in $\Delta v v d$ (Figure 3 , right panels) faithfully reproduced the period length and phase of all reporters and captured their phase delays compared with WT (Figure S1). The amplitudes of frq and $v v d$ oscillations in the dark were modeled quite well. In contrast the amplitude of the $\operatorname{csp} p-1$ oscillation was underestimated even more than in WT, supporting the notion (see 
above) that the csp-1 transcription could be additionally supported by an unknown activator. The expression dynamics of $v v d$ in LD cycles were fully captured, suggesting that Michaelis-Menten kinetics is suitable to quantitatively describe the activity of the $v v d$ promoter in WT and in $\Delta v v d$. The higher light-induced expression level of the $v v d$ reporter in $\Delta v v d$ compared with $W T$ was also captured by our model. Because the transcriptional output of the $v v d$ promoter is very sensitive to the activity of the light-activated WCC (Li et al., 2018), the agreement of our model with the $\Delta v v d$ and $W T$ data justifies our differentiation of various light-activated WCC species $\left(\right.$ WCC $^{*}$, WCC $^{*}-W_{C C}^{*}$ and WCC $\left.^{*} V V^{*}\right)$ and the rates of their interconversion. Our model did, however, not capture the complexity of the expression profile of frq in light-dark cycles, indicating that Michaelis-Menten-like promoter activation was not sufficient to describe the response of the frq promoter to light cues, in particular in $\Delta v v d$.

Specifically, in $\Delta v v d$, our Michaelis-Menten-based model of the frq promoter produced almost square-waves of frq expression in LD with a rather slow decline after light was turned off. In contrast, the luciferase measurements revealed an initial overshoot of frq expression after light on, followed by rapid adaptation of frq in continuous light and then a transient increase in frq expression after the LD transition. As discussed above, the complex transcription dynamics reflect the previously reported partial repression of the light-activated frq promoter (Cesbron et al., 2015; Li et al., 2018). This particular feature of the frq promoter has not yet been included in our molecular model scheme as the underlying mechanism is still awaiting identification and functional characterization of the components involved. However, it is only the acquisition of high-resolution data that made it possible to reveal the discrepancy between model and data and thus predict that the transcriptional dynamics of the frq promoter in response to light cannot be modeled by simple Michaelis-Menten-like promoter activation. Such prediction is not possible with generic models. 
Expression of $c s p-1$ in the $\Delta v v d$ background increased sharply after light on and then adapted rapidly, reflecting the negative feedback of CSP-1 on its own transcription. The dynamics of the CSP-1 feedback, which was captured by the model in $W T$, was underestimated in $\Delta v v d$, indicating that the model did not quantitatively describe the enhanced light-induced expression of CSP-1 in absence of VVD. Potentially, Michaelis-Menten-like promoter regulation is not sufficient to describe the dynamics of the $\operatorname{csp}-1$ promoter.

We then challenged the model by simulating 10 consecutive light-dark cycles (Figure S2, left panels). The system responded in the same manner to each of the LD cycles. None of the components was depleted or accumulated to higher levels over the time period, indicating that the system was balanced.

In constant darkness the expression levels of all IUCPEST reporters oscillated but showed dampening over time (see Figure 2). Our model was trained on the dampening bioluminescence oscillations in the dark it reproduced the dampening (Figure 3). Indeed, simulating prolonged incubation in the dark led to a substantial loss of amplitude (Figure S2, right panels). Since we have not allowed the possibility of desynchronization of individual oscillators in our analytical model, the damping in our model is due exclusively to a gradual loss of amplitude. However, it is possible that the experimentally observed dampening is due to desynchronization and that the modelled loss of amplitude does not reflect a physiological relevant process. Indeed, loss of amplitude was prevented when the dephosphorylation rate of WCC, i.e. its reactivation, was slightly increased (Figure S3).

Overall, however, the model almost quantitatively predicted the transcriptional dynamics of two hierarchical levels of the circadian clock in the dark and in lightdark cycles in WT. The model was generally less precise in predicting transcription rhythms and dynamics in $\Delta v v d$.

The detailed data-based model allowed us to ask specific questions that cannot be addressed with a generic clock model. For example, previous models of the 
Neurospora circadian clock used Hill functions which was crucial for robust circadian oscillation of frq transcription (Bellman et al., 2018; Dovzhenok et al., 2015; Leloup et al., 1999; Liu et al., 2019; Ruoff and Rensing, 1996). As we did not introduce a Hill-function for the transcriptional production of frq, the circadian oscillation in our model depends critically on the delay between the synthesis of frq and the appearance of the fully assembled and active FFC. This process, which was modeled by FRQ translation and six generic maturation steps, introduced a delay in the accumulation of active FFC and supported circadian oscillation of frq. Shortening the delay by successive removal of maturation steps resulted in increasing expression levels of frq and arrhythmicity (Figure 4A). Thus, our model suggests that maturation of the FFC may be an important process that should be studied experimentally. Indeed, this aspect is still very poorly understood, and we do not know how, when, in what order, and in what cellular compartments FRQ dimerizes, assembles with $\mathrm{FRH}$ and $\mathrm{CK} 1 \mathrm{a}$, and if $\mathrm{FRQ}$ requires licensing phosphorylation somewhere along this pathway to become an active inhibitor of WCC in the nucleus.

Our model also predicts that only a fraction of the newly synthesized $\mathrm{FRQ}$ assembles with $\mathrm{FRH}$ while the majority is degraded. Due to the slow assembly process unassembled and partially assembled FRQ is more abundant than fully assembled, active FFC at any given time (Figure 4B). The high fraction of partially assembled $F R Q$ species could explain the substoichiometic levels of $F R H$ that are found in complex with total FRQ in vivo (Hurley et al., 2013), although one molecule of $F R Q$ is capable of binding one molecule of $F R H$ (Lauinger et., 2014).

The continuous recording of promoter-specific luciferase reporters in vivo in dark and light made it possible to estimate the intrinsic firing rate of the frq and $v v d$ promoters $\left(V_{\max }\right)$ and to uncover functional differences in promoter architecture. Previous quantitative ChIP-PCR analyses revealed a similar affinity of the lightactivated WCC for the LREs of frq and vvd (Cesbron et al., 2015; Li et al., 2018). Yet, our model predicted a small $\mathrm{K}_{\mathrm{M}}$ for WCC-dependent transcription activation of frq and a larger $\mathrm{K}_{\mathrm{M}}$ for $v v d$. The data indicate that the affinity of the transcription 
factor for its LRE does not directly correlate with gene transcription. The difference likely reflects that the frq LRE is located in the core promoter such that bound $W_{C C}{ }^{*} W_{W C}^{*}$ can directly activate the core promoter. In contrast, the $v v d$ LRE is located in an upstream enhancer region. Hence, activation of $v v d$ transcription is dependent on looping of the LRE-bound $W_{C C}{ }^{*} W W C^{*}$ to the core promoter. The coupled equilibria of TF-binding to the remote LRE and looping of the LRE-bound TF to the promoter result in an overall higher $\mathrm{K}_{\mathrm{M}}$ for the activation of transcription at the $v v d$ promoter (Li et al., 2018). Furthermore, our model predicted a small Vmax for frq, consistent with frq being a weak promoter with an intrinsically low firing rate. The light-activated $v v d$ promoter has a high intrinsic firing rate, consistent with a large predicted $V_{\max }$. Our simulations with these promoter-specific parameters (Figure 5A, B) captured in principle the previously reported saturation of frq transcription at rather low light intensity while $v v d$ responds over a much wider range proportional to the intensity of light (Cesbron et al., 2015; Li et al., 2018).

In summary, the luc reporter strains allowed measuring transcript levels and dynamics with high temporal resolution. Obtaining such comprehensive data justified the construction of a detailed molecular model of the Neurospora circadian system. The wiring scheme of the model and the derived kinetic parameters captured crucial features of the circadian system, but cannot yet accurately predict the temporal dynamics of all processes. This discrepancy led to new testable hypotheses. Overall, therefore, we believe that data-based approaches to circadian clocks as the one presented here can become a valuable tool for quantitatively understanding the interaction of the molecular clock components.

\section{Acknowledgements}

The authors gratefully acknowledge the data storage service SDS@ hd supported by the Ministry of Science, Research and the Arts Baden-Württemberg (MWK) and 
the German Research Foundation (DFG) through grant INST 35/1314-1 FUGG and INST 35/1503-1 FUGG. M.B. and T.H. are supported by the Deutsche Forschungsgemeinschaft, TRR186.

Author Contributions: M.B. and T.H. conceived the study. A.S. and C.L. performed the modelling analysis. A.C.R.D. carried out the experiments. A.S., M.B. wrote the paper.

\section{Conflict of interest}

The authors declare that they have no conflict of interest.

\section{References}

Akman, O.E., Locke, J.C., Tang, S., Carre, I., Millar, A.J., and Rand, D.A. (2008). Isoform switching facilitates period control in the Neurospora crassa circadian clock. Mol Syst Biol 4, 164. 10.1038/msb.2008.5.

Arcak, M., and Sontag, E.D. (2006). Diagonal stability of a class of cyclic systems and its connection with the secant criterion. Automatica 42, 1531-1537. https://doi.org/10.1016/j.automatica.2006.04.009.

Aronson, B.D., Johnson, K.A., Loros, J.J., and Dunlap, J.C. (1994). Negative feedback defining a circadian clock: autoregulation of the clock gene frequency. Science 263, 1578-1584. 10.1126/science.8128244.

Aschoff, J. (1965). Circadian Rhythms in Man. Science 148, 1427-1432. 10.1126/science.148.3676.1427.

Bass, J., and Lazar, M.A. (2016). Circadian time signatures of fitness and disease. Science 354, 994-999. 10.1126/science.aah4965.

Bechtold, D.A., Gibbs, J.E., and Loudon, A.S. (2010). Circadian dysfunction in disease. Trends Pharmacol Sci 31, 191-198. 10.1016/j.tips.2010.01.002.

Belden, W.J., Larrondo, L.F., Froehlich, A.C., Shi, M., Chen, C.H., Loros, J.J., and Dunlap, J.C. (2007). The band mutation in Neurospora crassa is a dominant allele of ras-1 implicating RAS signaling in circadian output. Genes Dev 21, 1494-1505. 10.1101/gad.1551707.

Bellman, J., Kim, J.K., Lim, S., and Hong, C.I. (2018). Modeling Reveals a Key Mechanism for Light-Dependent Phase Shifts of Neurospora Circadian Rhythms. Biophys J 115, 1093-1102. 10.1016/j.bpj.2018.07.029.

Burt, P., Grabe, S., Madeti, C., Upadhyay, A., Merrow, M., Roenneberg, T., ... \& Schmal, C. (2021). Principles underlying the complex dynamics of temperature entrainment by a circadian clock. Iscience, 24(11), 103370. https://doi.org/10.1016/j.isci.2021.103370 
Cesbron, F., Brunner, M., and Diernfellner, A.C. (2013). Light-dependent and circadian transcription dynamics in vivo recorded with a destabilized luciferase reporter in Neurospora. PLoS One 8, e83660. 10.1371/journal.pone.0083660.

Cesbron, F., Oehler, M., Ha, N., Sancar, G., and Brunner, M. (2015). Transcriptional refractoriness is dependent on core promoter architecture. Nat Commun 6, 6753. 10.1038/ncomms7753.

Cha, J., Chang, S.S., Huang, G., Cheng, P., and Liu, Y. (2008). Control of WHITE COLLAR localization by phosphorylation is a critical step in the circadian negative feedback process. EMBO J 27, 3246-3255. 10.1038/emboj.2008.245.

Cha, J., Yuan, H., and Liu, Y. (2011). Regulation of the activity and cellular localization of the circadian clock protein FRQ. J Biol Chem 286, 11469-11478. 10.1074/jbc.M111.219782.

Challet, E. (2007). [Clock genes, circadian rhythms and food intake]. Pathol Biol (Paris) 55, 176-177. 10.1016/j.patbio.2006.12.005.

Chen, C.H., Ringelberg, C.S., Gross, R.H., Dunlap, J.C., and Loros, J.J. (2009). Genome-wide analysis of light-inducible responses reveals hierarchical light signalling in Neurospora. EMBO J 28, 1029-1042. 10.1038/emboj.2009.54.

Cheng, P., He, Q., He, Q., Wang, L., and Liu, Y. (2005). Regulation of the Neurospora circadian clock by an RNA helicase. Genes Dev 19, 234-241. 10.1101/gad.1266805.

Cheng, P., He, Q., Yang, Y., Wang, L., and Liu, Y. (2003). Functional conservation of light, oxygen, or voltage domains in light sensing. Proc Natl Acad Sci U S A 100, 5938-5943. 10.1073/pnas.1031791100.

Cheng, P., Yang, Y., Heintzen, C., and Liu, Y. (2001a). Coiled-coil domainmediated FRQ-FRQ interaction is essential for its circadian clock function in Neurospora. EMBO J 20, 101-108. 10.1093/emboj/20.1.101.

Cheng, P., Yang, Y., and Liu, Y. (2001b). Interlocked feedback loops contribute to the robustness of the Neurospora circadian clock. Proc Natl Acad Sci U S A 98, 7408-7413. 10.1073/pnas.121170298.

Corrochano, L.M. (2007). Fungal photoreceptors: sensory molecules for fungal development and behaviour. Photochem Photobiol Sci 6, 725-736. 10.1039/b702155k.

Crosthwaite, S.K., Dunlap, J.C., and Loros, J.J. (1997). Neurospora wc-1 and wc2: transcription, photoresponses, and the origins of circadian rhythmicity. Science 276, 763-769. 10.1126/science.276.5313.763.

Demarsy, E., and Fankhauser, C. (2009). Higher plants use LOV to perceive blue light. Curr Opin Plant Biol 12, 69-74. 10.1016/j.pbi.2008.09.002.

Denault, D.L., Loros, J.J., and Dunlap, J.C. (2001). WC-2 mediates WC-1-FRQ interaction within the PAS protein-linked circadian feedback loop of Neurospora. EMBO J 20, 109-117. 10.1093/emboj/20.1.109.

Diernfellner, A.C.R., and Brunner, M. (2020). Phosphorylation Timers in the Neurospora crassa Circadian Clock. J Mol Biol 432, 3449-3465. 10.1016/j.jmb.2020.04.004. 
Dovzhenok, A.A., Baek, M., Lim, S., and Hong, C.I. (2015). Mathematical modeling and validation of glucose compensation of the neurospora circadian clock. Biophys J 108, 1830-1839. 10.1016/j.bpj.2015.01.043.

Dunlap, J.C. (1999). Molecular bases for circadian clocks. Cell 96, 271-290. 10.1016/s0092-8674(00)80566-8.

Elvin, M., Loros, J.J., Dunlap, J.C., and Heintzen, C. (2005). The PAS/LOV protein VIVID supports a rapidly dampened daytime oscillator that facilitates entrainment of the Neurospora circadian clock. Genes Dev 19, 2593-2605. 10.1101/gad.349305.

Forger, D.B. (2011). Signal processing in cellular clocks. Proc Natl Acad Sci U S A 108, 4281-4285. 10.1073/pnas.1004720108.

Francois, P. (2005). A model for the Neurospora circadian clock. Biophys J 88, 2369-2383. 10.1529/biophysj.104.053975.

Froehlich, A.C., Liu, Y., Loros, J.J., and Dunlap, J.C. (2002). White Collar-1, a circadian blue light photoreceptor, binding to the frequency promoter. Science 297, 815-819. 10.1126/science.1073681.

Gin, E., Diernfellner, A.C., Brunner, M., and Hofer, T. (2013). The Neurospora photoreceptor VIVID exerts negative and positive control on light sensing to achieve adaptation. Mol Syst Biol 9, 667. 10.1038/msb.2013.24.

Goldbeter, A. (1995). A model for circadian oscillations in the Drosophila period protein (PER). Proc Biol Sci 261, 319-324. 10.1098/rspb.1995.0153.

Gorl, M., Merrow, M., Huttner, B., Johnson, J., Roenneberg, T., and Brunner, M. (2001). A PEST-like element in FREQUENCY determines the length of the circadian period in Neurospora crassa. EMBO J 20, 7074-7084. 10.1093/emboj/20.24.7074.

Guo, J., Cheng, P., and Liu, Y. (2010). Functional significance of FRH in regulating the phosphorylation and stability of Neurospora circadian clock protein FRQ. J Biol Chem 285, 11508-11515. 10.1074/jbc.M109.071688.

Harding, R.W., and Turner, R.V. (1981). Photoregulation of the Carotenoid Biosynthetic Pathway in Albino and White Collar Mutants of Neurospora crassa. Plant Physiol 68, 745-749. 10.1104/pp.68.3.745.

He, Q., Cheng, P., Yang, Y., Wang, L., Gardner, K.H., and Liu, Y. (2002). White collar-1, a DNA binding transcription factor and a light sensor. Science 297, 840843. 10.1126/science.1072795.

Heintzen, C., Loros, J.J., and Dunlap, J.C. (2001). The PAS protein VIVID defines a clock-associated feedback loop that represses light input, modulates gating, and regulates clock resetting. Cell 104, 453-464. 10.1016/s0092-8674(01)00232-x. Hellweger, F.L. (2010). Resonating circadian clocks enhance fitness in cyanobacteria in silico. Ecological Modelling 221, 1620-1629. https://doi.org/10.1016/j.ecolmodel.2010.03.015.

Hong, C.I., Jolma, I.W., Loros, J.J., Dunlap, J.C., and Ruoff, P. (2008). Simulating dark expressions and interactions of frq and wc-1 in the Neurospora circadian clock. Biophys J 94, 1221-1232. 10.1529/biophysj.107.115154. 
Huang, G., Chen, S., Li, S., Cha, J., Long, C., Li, L., He, Q., and Liu, Y. (2007). Protein kinase $A$ and casein kinases mediate sequential phosphorylation events in the circadian negative feedback loop. Genes Dev 21, 3283-3295. $10.1101 /$ gad.1610207.

Hurley, Jennifer M., et al. "Conserved RNA helicase FRH acts nonenzymatically to support the intrinsically disordered Neurospora clock protein FRQ." Molecular cell 52.6 (2013): 832-843.

Hunt, S.M., Elvin, M., Crosthwaite, S.K., and Heintzen, C. (2007). The PAS/LOV protein VIVID controls temperature compensation of circadian clock phase and development in Neurospora crassa. Genes Dev 21, 1964-1974. 10.1101/gad.437107.

Kaldi, K., Gonzalez, B.H., and Brunner, M. (2006). Transcriptional regulation of the Neurospora circadian clock gene wc-1 affects the phase of circadian output. EMBO Rep 7, 199-204. 10.1038/sj.embor.7400595.

Larrondo, L.F., Olivares-Yanez, C., Baker, C.L., Loros, J.J., and Dunlap, J.C. (2015). Circadian rhythms. Decoupling circadian clock protein turnover from circadian period determination. Science 347, 1257277. 10.1126/science.1257277. Lauter, F.R., and Russo, V.E. (1991). Blue light induction of conidiation-specific genes in Neurospora crassa. Nucleic Acids Res 19, 6883-6886. 10.1093/nar/19.24.6883.

Lauinger, Linda, et al. "The RNA helicase FRH is an ATP-dependent regulator of CK1a in the circadian clock of Neurospora crassa." Nature communications 5.1 (2014): 1-10.

Lee, K., Loros, J.J., and Dunlap, J.C. (2000). Interconnected feedback loops in the Neurospora circadian system. Science 289, 107-110. 10.1126/science.289.5476.107.

Leloup, J.C., Gonze, D., and Goldbeter, A. (1999). Limit cycle models for circadian rhythms based on transcriptional regulation in Drosophila and Neurospora. J Biol Rhythms 14, 433-448. 10.1177/074873099129000948.

Li, C., Cesbron, F., Oehler, M., Brunner, M., and Hofer, T. (2018). Frequency Modulation of Transcriptional Bursting Enables Sensitive and Rapid Gene Regulation. Cell Syst 6, 409-423 e411. 10.1016/j.cels.2018.01.012.

Linden, H., and Macino, G. (1997). White collar 2, a partner in blue-light signal transduction, controlling expression of light-regulated genes in Neurospora crassa. EMBO J 16, 98-109. 10.1093/emboj/16.1.98.

Liu, X., Chen, A., Caicedo-Casso, A., Cui, G., Du, M., He, Q., Lim, S., Kim, H.J., Hong, C.I., and Liu, Y. (2019). FRQ-CK1 interaction determines the period of circadian rhythms in Neurospora. Nat Commun 10, 4352. 10.1038/s41467-01912239-w.

Lowrey, P.L., and Takahashi, J.S. (2000). Genetics of the mammalian circadian system: Photic entrainment, circadian pacemaker mechanisms, and 
posttranslational regulation. Annu Rev Genet 34, 533-562. 10.1146/annurev.genet.34.1.533.

Malzahn, E., Ciprianidis, S., Kaldi, K., Schafmeier, T., and Brunner, M. (2010). Photoadaptation in Neurospora by competitive interaction of activating and inhibitory LOV domains. Cell 142, 762-772. 10.1016/j.cell.2010.08.010.

Mehra, A., Shi, M., Baker, C.L., Colot, H.V., Loros, J.J., and Dunlap, J.C. (2009). A role for casein kinase 2 in the mechanism underlying circadian temperature compensation. Cell 137, 749-760. 10.1016/j.cell.2009.03.019.

Neiss, A., Schafmeier, T., and Brunner, M. (2008). Transcriptional regulation and function of the Neurospora clock gene white collar 2 and its isoforms. EMBO Rep 9, 788-794. 10.1038/embor.2008.113.

Ouyang, Y., Andersson, C.R., Kondo, T., Golden, S.S., and Johnson, C.H. (1998). Resonating circadian clocks enhance fitness in cyanobacteria. Proc Natl Acad Sci U S A 95, 8660-8664. 10.1073/pnas.95.15.8660.

Pittendrigh, C.S. (1954). On Temperature Independence in the Clock System Controlling Emergence Time in Drosophila. Proc Natl Acad Sci U S A 40, 10181029. 10.1073/pnas.40.10.1018.

Pittendrigh, C.S. (1960). Circadian rhythms and the circadian organization of living systems. Cold Spring Harb Symp Quant Biol 25, 159-184. 10.1101/sqb.1960.025.01.015.

Ponting, C.P., and Aravind, L. (1997). PAS: a multifunctional domain family comes to light. Curr Biol 7, R674-677. 10.1016/s0960-9822(06)00352-6.

Querfurth, C., Diernfellner, A., Heise, F., Lauinger, L., Neiss, A., Tataroglu, O., Brunner, M., and Schafmeier, T. (2007). Posttranslational regulation of Neurospora circadian clock by CK1a-dependent phosphorylation. Cold Spring Harb Symp Quant Biol 72, 177-183. 10.1101/sqb.2007.72.025.

Raue, A., Steiert, B., Schelker, M., Kreutz, C., Maiwald, T., Hass, H., Vanlier, J., Tonsing, C., Adlung, L., Engesser, R., et al. (2015). Data2Dynamics: a modeling environment tailored to parameter estimation in dynamical systems. Bioinformatics 31, 3558-3560. 10.1093/bioinformatics/btv405.

Ruger-Herreros, C., Gil-Sanchez Mdel, M., Sancar, G., Brunner, M., and Corrochano, L.M. (2014). Alteration of light-dependent gene regulation by the absence of the $\mathrm{RCO}-1 / \mathrm{RCM}-1$ repressor complex in the fungus Neurospora crassa. PLoS One 9, e95069. 10.1371/journal.pone.0095069.

Ruoff, P., Mohsenzadeh, S., and Rensing, L. (1996). Circadian rhythms and protein turnover: the effect of temperature on the period lengths of clock mutants simulated by the Goodwin oscillator. Naturwissenschaften 83, 514-517. 10.1007/BF01141953.

Ruoff, P., and Rensing, L. (1996). The Temperature-Compensated Goodwin Model Simulates Many Circadian Clock Properties. Journal of Theoretical Biology 179, 275-285.

Sancar, C., Sancar, G., Ha, N., Cesbron, F., and Brunner, M. (2015). Dawn- and dusk-phased circadian transcription rhythms coordinate anabolic and catabolic functions in Neurospora. BMC Biol 13, 17. 10.1186/s12915-015-0126-4. 
Sancar, G., Sancar, C., Brugger, B., Ha, N., Sachsenheimer, T., Gin, E., Wdowik, S., Lohmann, I., Wieland, F., Hofer, T., et al. (2011). A global circadian repressor controls antiphasic expression of metabolic genes in Neurospora. Mol Cell 44, 687697. 10.1016/j.molcel.2011.10.019.

Schafmeier, T., Diernfellner, A., Schafer, A., Dintsis, O., Neiss, A., and Brunner, M. (2008). Circadian activity and abundance rhythms of the Neurospora clock transcription factor WCC associated with rapid nucleo-cytoplasmic shuttling. Genes Dev 22, 3397-3402. 10.1101/gad.507408.

Schafmeier, T., and Diernfellner, A.C. (2011). Light input and processing in the circadian clock of Neurospora. FEBS Lett 585, 1467-1473. 10.1016/j.febslet.2011.03.050.

Schafmeier, T., Haase, A., Kaldi, K., Scholz, J., Fuchs, M., and Brunner, M. (2005). Transcriptional feedback of Neurospora circadian clock gene by phosphorylationdependent inactivation of its transcription factor. Cell 122, 235-246. 10.1016/j.cell.2005.05.032.

Shi, M., Collett, M., Loros, J.J., and Dunlap, J.C. (2010). FRQ-interacting RNA helicase mediates negative and positive feedback in the Neurospora circadian clock. Genetics 184, 351-361. 10.1534/genetics.109.111393.

Smith, K.M., Sancar, G., Dekhang, R., Sullivan, C.M., Li, S., Tag, A.G., Sancar, C., Bredeweg, E.L., Priest, H.D., McCormick, R.F., et al. (2010). Transcription factors in light and circadian clock signaling networks revealed by genomewide mapping of direct targets for neurospora white collar complex. Eukaryot Cell 9, 1549-1556. 10.1128/EC.00154-10.

Suetsugu, N., and Wada, M. (2013). Evolution of three LOV blue light receptor families in green plants and photosynthetic stramenopiles: phototropin, ZTL/FKF1/LKP2 and aureochrome. Plant Cell Physiol 54, 8-23. 10.1093/pcp/pcs165.

Talora, C., Franchi, L., Linden, H., Ballario, P., and Macino, G. (1999). Role of a white collar-1-white collar-2 complex in blue-light signal transduction. EMBO J 18, 4961-4968. 10.1093/emboj/18.18.4961.

Thron, C.D. (1991). The secant condition for instability in biochemical feedback control-I. The role of cooperativity and saturability. Bulletin of Mathematical Biology 53, 383-401. https://doi.org/10.1016/S0092-8240(05)80394-5.

Tseng, Y.Y., Hunt, S.M., Heintzen, C., Crosthwaite, S.K., and Schwartz, J.M. (2012). Comprehensive modelling of the Neurospora circadian clock and its temperature compensation. PLoS Comput Biol 8, e1002437. 10.1371/journal.pcbi.1002437.

Upadhyay, A., Brunner, M., and Herzel, H. (2019). An Inactivation Switch Enables Rhythms in a Neurospora Clock Model. Int J Mol Sci 20. 10.3390/ijms20122985. Upadhyay, A., Marzoll, D., Diernfellner, A., Brunner, M., \& Herzel, H. (2020). Multiple random phosphorylations in clock proteins provide long delays and switches. Scientific reports, 10(1), 1-13. https://doi.org/10.1038/s41598-02079277-z 
Yang, Y., Cheng, P., He, Q., Wang, L., and Liu, Y. (2003). Phosphorylation of FREQUENCY protein by casein kinase II is necessary for the function of the Neurospora circadian clock. Mol Cell Biol 23, 6221-6228. 10.1128/mcb.23.17.6221-6228.2003.

Yang, Y., Cheng, P., and Liu, Y. (2002). Regulation of the Neurospora circadian clock by casein kinase II. Genes Dev 16, 994-1006. 10.1101/gad.965102.

Yang, Y., Cheng, P., Zhi, G., and Liu, Y. (2001). Identification of a calcium/calmodulin-dependent protein kinase that phosphorylates the Neurospora circadian clock protein FREQUENCY. J Biol Chem 276, 41064-41072. 10.1074/jbc.M106905200.

Young, M.W., and Kay, S.A. (2001). Time zones: a comparative genetics of circadian clocks. Nat Rev Genet 2, 702-715. 10.1038/35088576.

Zoltowski, B.D., and Crane, B.R. (2008). Light activation of the LOV protein vivid generates a rapidly exchanging dimer. Biochemistry 47, 7012-7019. $10.1021 /$ bi8007017. 


\section{Materials and methods}

\section{Neurospora strains and plasmids}

The Neurospora strains denoted with $W T$ and $\Delta v v d$ carried the ras $1^{\text {bd }}$ mutation (Belden et al., 2007) and either a pfrq-IucPEST, pvvd-lucPEST (Cesbron et al., 2013), pfam3-IucPEST, formerly called desat-lucPEST (Sancar et al., 2011) or a pcsp1-IucPEST reporter gene integrated downstream of the his-3 locus. For pcsp1-IucPEST, a 7395bp fragment immediately upstream of the csp-1 ORF was amplified from gDNA and cloned in front of the lucPEST ORF using EcoRI (vector)/Mfel(PCR insert) and Notl restriction sites. The resulting plasmid was used to transform the above mentioned Neurospora strains.

\section{Real-time luciferase activity measurements}

Sorbose medium containing 1x FGS (0.05 \%fructose, 0.05\% glucose, 2\% sorbose), $1 \times$ Vogels, $1 \%$ agarose, $10 \mathrm{ng} / \mathrm{ml}$ biotin, and $25 \mu \mathrm{M}$ firefly Luciferin was used for the assessment of the luciferase activity. 96-well plates were inoculated with $3 \times 10^{4}$ conidia per well and incubated in $\mathrm{DD}$ at $25^{\circ} \mathrm{C}$. Bioluminescence was recorded in DD or LD at $25^{\circ} \mathrm{C}$ with EnSpire Multilabel Readers (Perkin Elmer). The light intensity was $0.25 \mu \mathrm{E}$. Three independent experiments with multiple biological replicates each were performed to generate the data $(n \geq 30)$.

\section{Mathematical modelling and parameter estimation}

The reactions of the model were translated to ODEs

$$
\frac{d(X(t, \theta))}{d t}=f_{x}(X(t, \theta), u(t), \theta)
$$

where $\boldsymbol{\theta}$ is a parameter $\boldsymbol{\theta}=\left(\boldsymbol{\theta}_{1}, \boldsymbol{\theta}_{2} . . \boldsymbol{\theta}_{\boldsymbol{i}}\right)$. The initial state of the system is described by $\boldsymbol{X}(\mathbf{0}, \boldsymbol{\theta})=\boldsymbol{f}_{X_{0}}(\boldsymbol{\theta})$ The variables $\boldsymbol{X}$ correspond to the dynamics of the concentration of molecular components of the model. To derive the unknown model parameters, the circadian model was calibrated by a maximum likelihood 
bioRxiv preprint doi: https://doi.org/10.1101/2022 01 24.477555; this version posted February 19, 2022. The copyright holder for this preprint

(which was not certified by peer review) is the author/funder, who has granted bioRxiv a license to display the preprint in perpetuity. It is made available under aCC-BY-NC-ND 4.0 International license.

estimation using quantitative experimental data obtained by luciferase measurements. The model was fitted to the luciferase data using (MATLAB version 2016b) D2D software package from http://www.data2dynamics.org (Raue et al. 2015). 

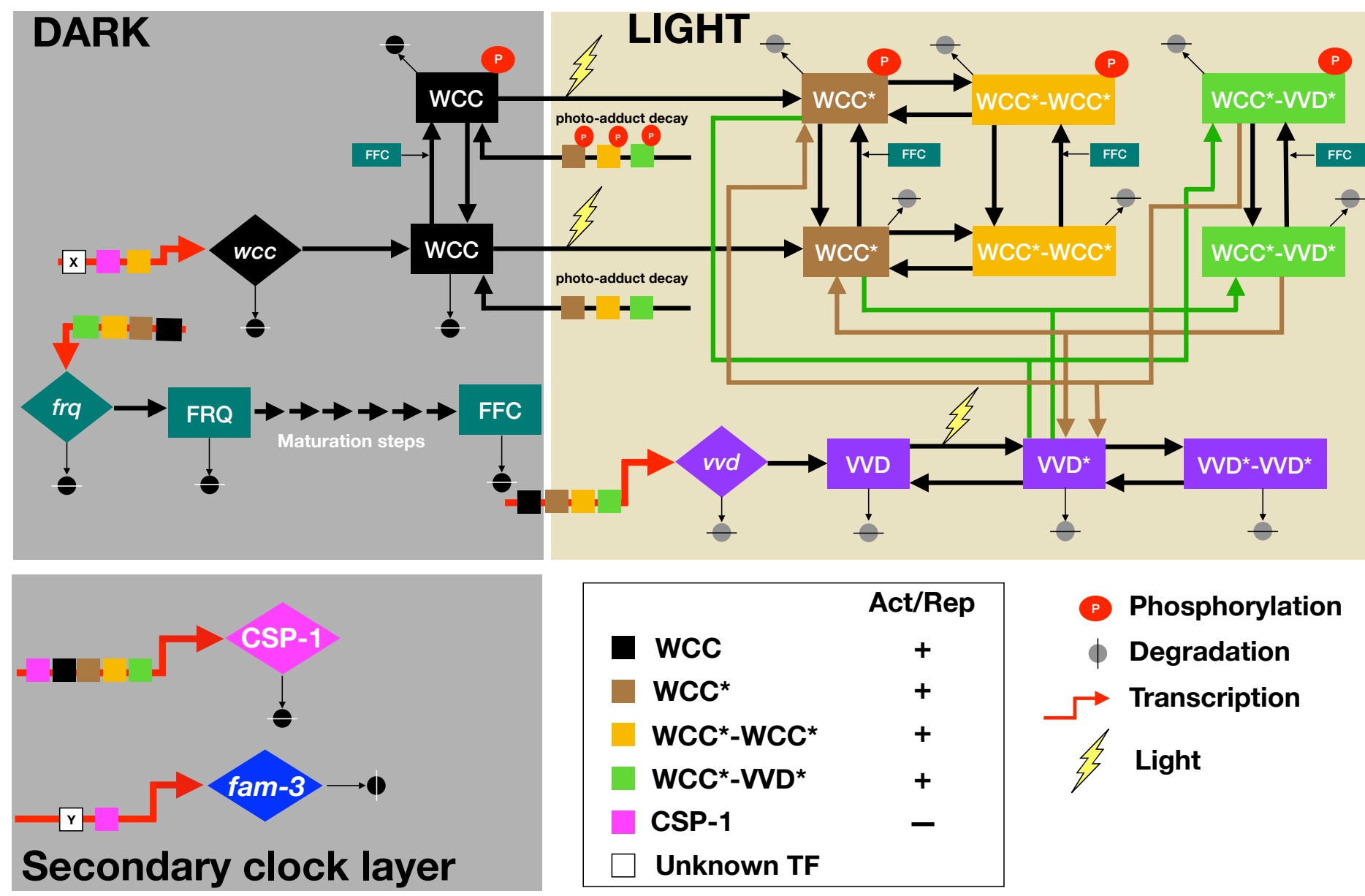
Fig. 1. Schematic of the Neurospora circadian clock in dark and light.

Thick red arrows indicate transcription, and the small colored square boxes on these arrows indicate the TFs controlling the respective gene. White boxes $X$ and $\mathrm{Y}$ indicate unidentified TFs activating transcription of $w c c$ and $c s p-1$, respectively. Large diamond and square boxes represent the indicated mRNA and protein species, respectively. $F R Q$, inactive Frequency protein; FFC, assembled, active FRQ-FRH-CK1a complex; WCC, White Collar Complex, VVD, Vivid; WCC* and $\mathrm{VVD}^{\star}$, light-activated species; csp-1, conidial separation 1; fam-3, fatty acid desaturase. 

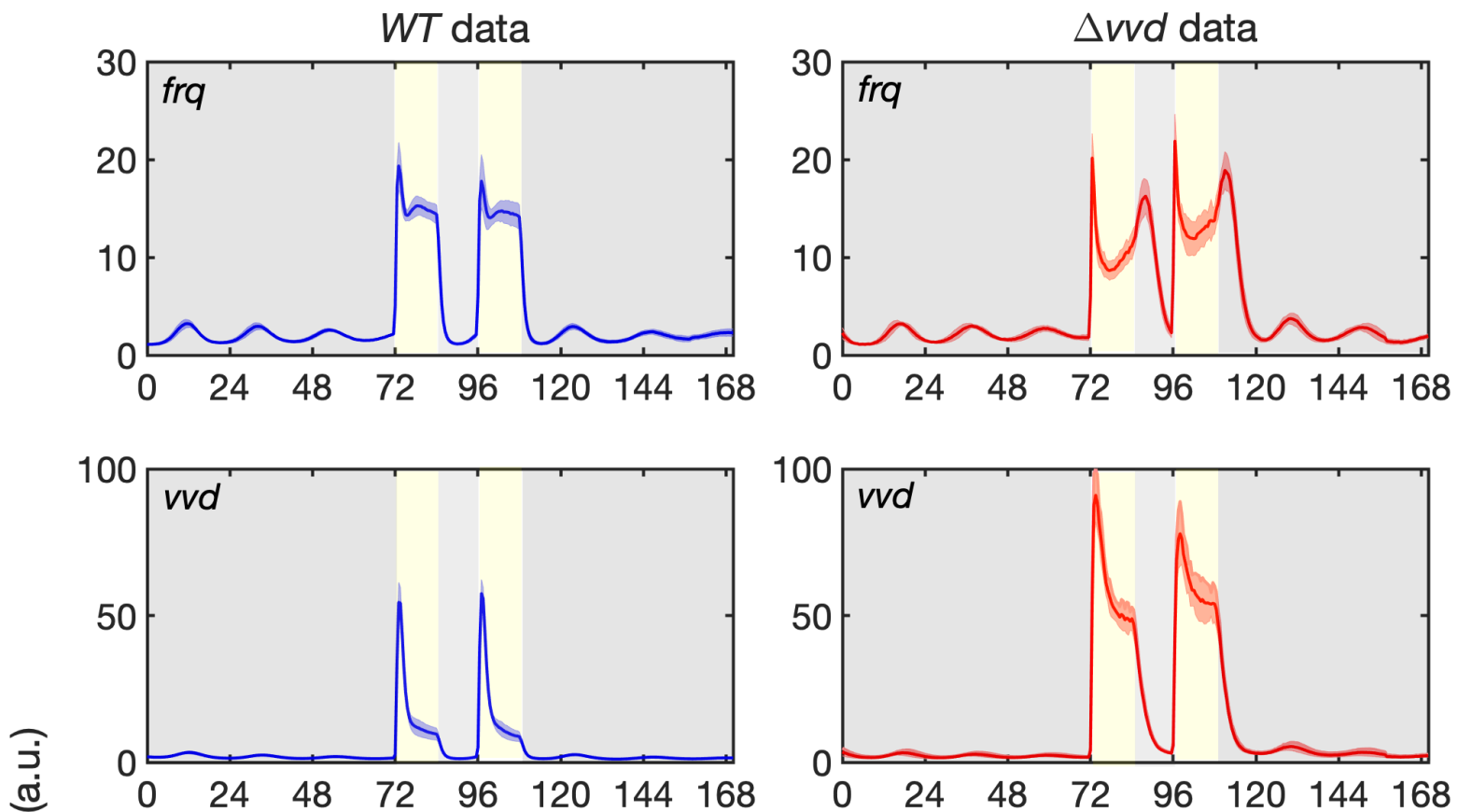

음
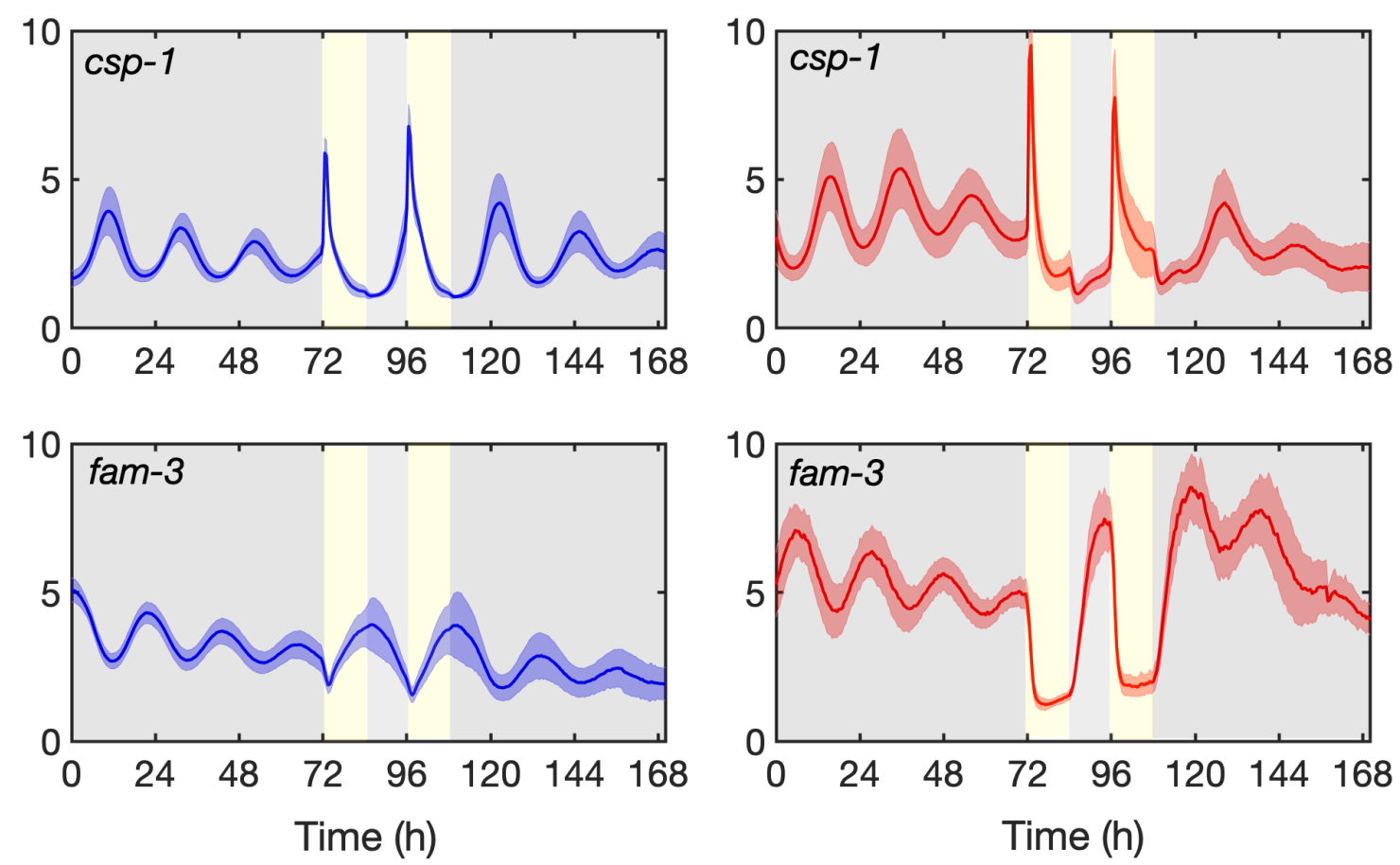

Figure 2 


\section{Fig. 2. Temporal expression profiles of reporter genes in DD and LD.}

Luciferase activity under the control of the indicated promoters in WT (left panels) and $\Delta v v d$ (right panels). The solid line represents the average of 30 measurements from 3 independent experiments. The shaded areas correspond to the standard deviation, SD. Yellow vertical boxes and grey areas indicate $12 \mathrm{~h}$ light periods and dark periods, respectively. 

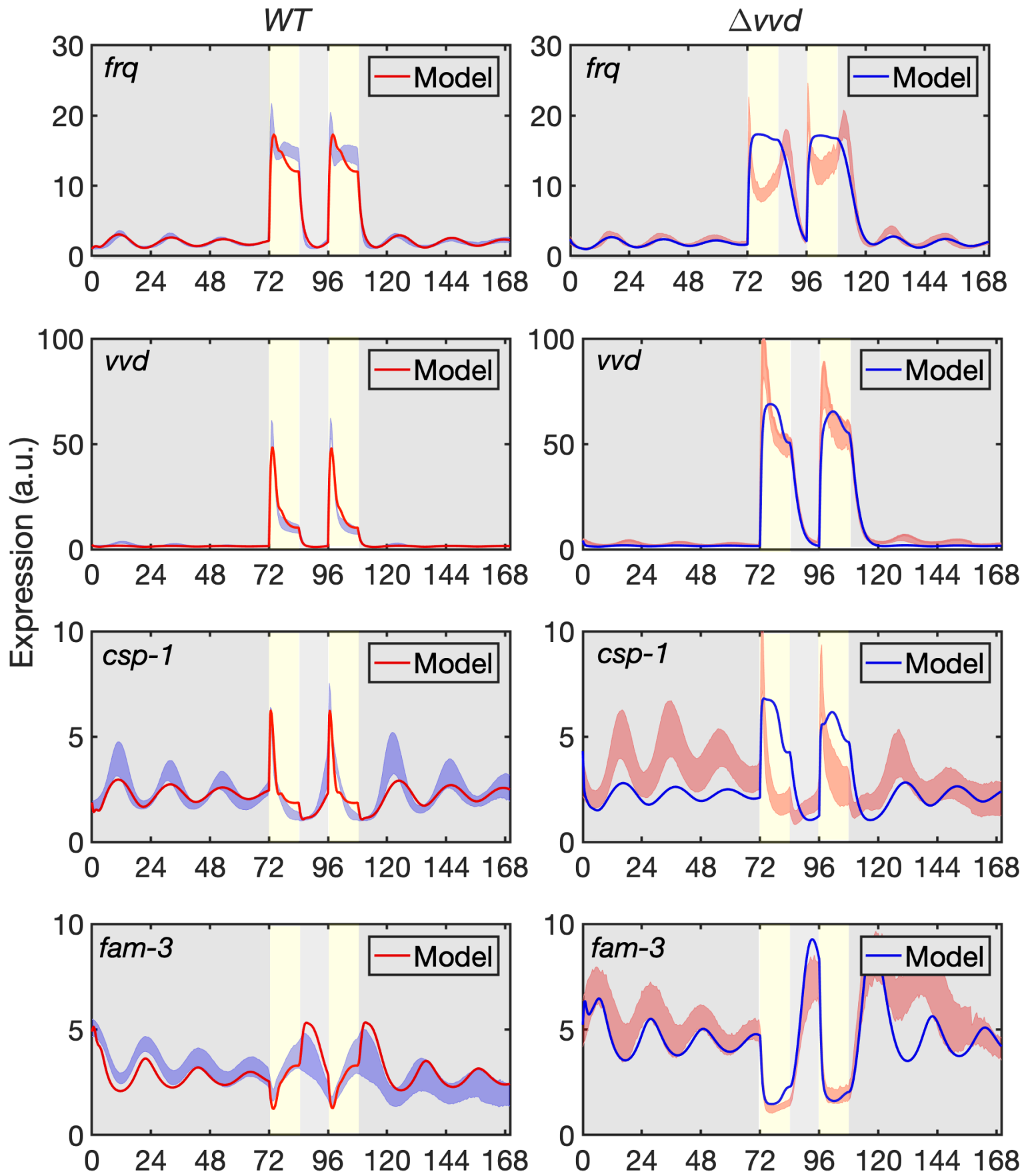

Time (h)

Time (h) 
Fig. 3. Model fitted to reporter gene expression in DD and LD.

Trajectories of the model (solid red lines in $W T$ and solid blue lines in $\Delta v v d$ ) fitted to the luciferase activity expressed under the control of the indicated promoters (standard deviation is shown, see Figure 2) in $W T$ (left panels) and $\Delta v v d$ (right panels). Yellow vertical boxes and grey areas indicate $12 \mathrm{~h}$ light periods and dark periods, respectively. 
A
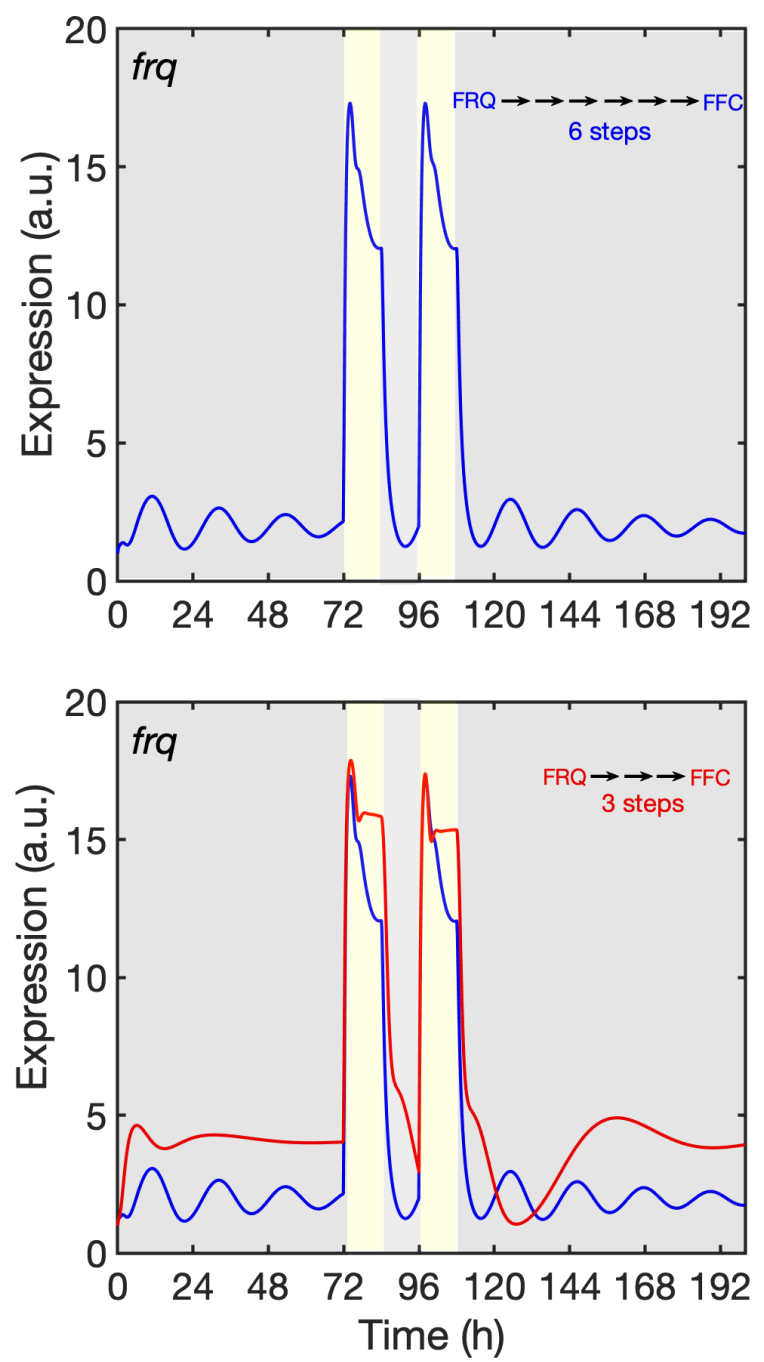

B

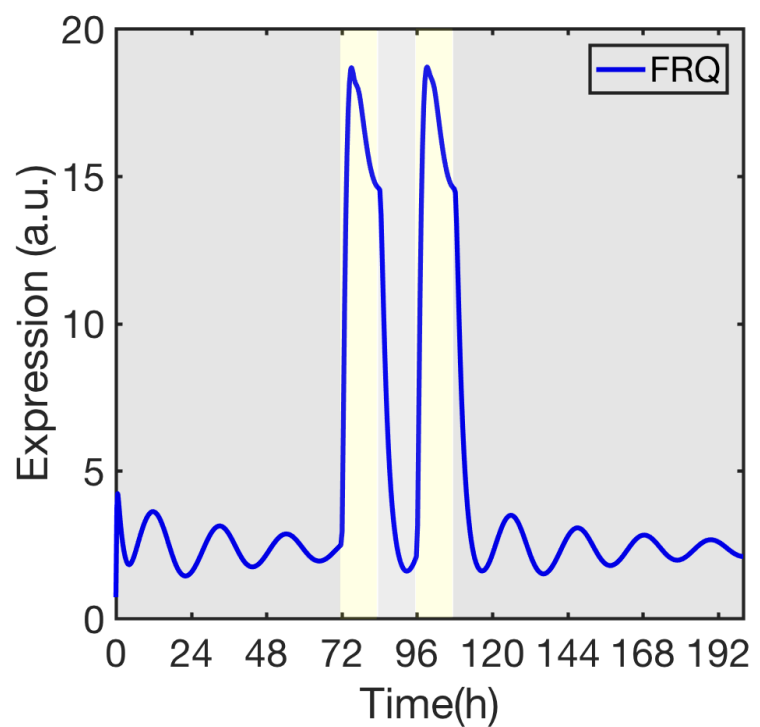

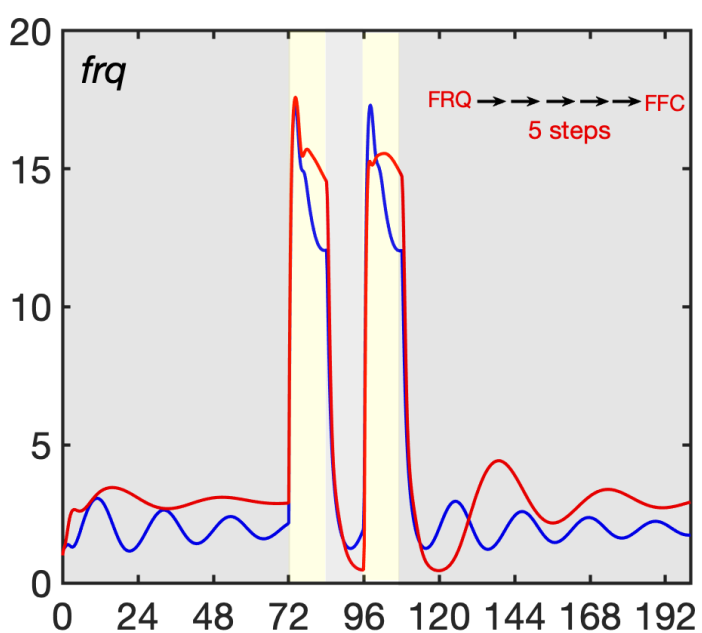
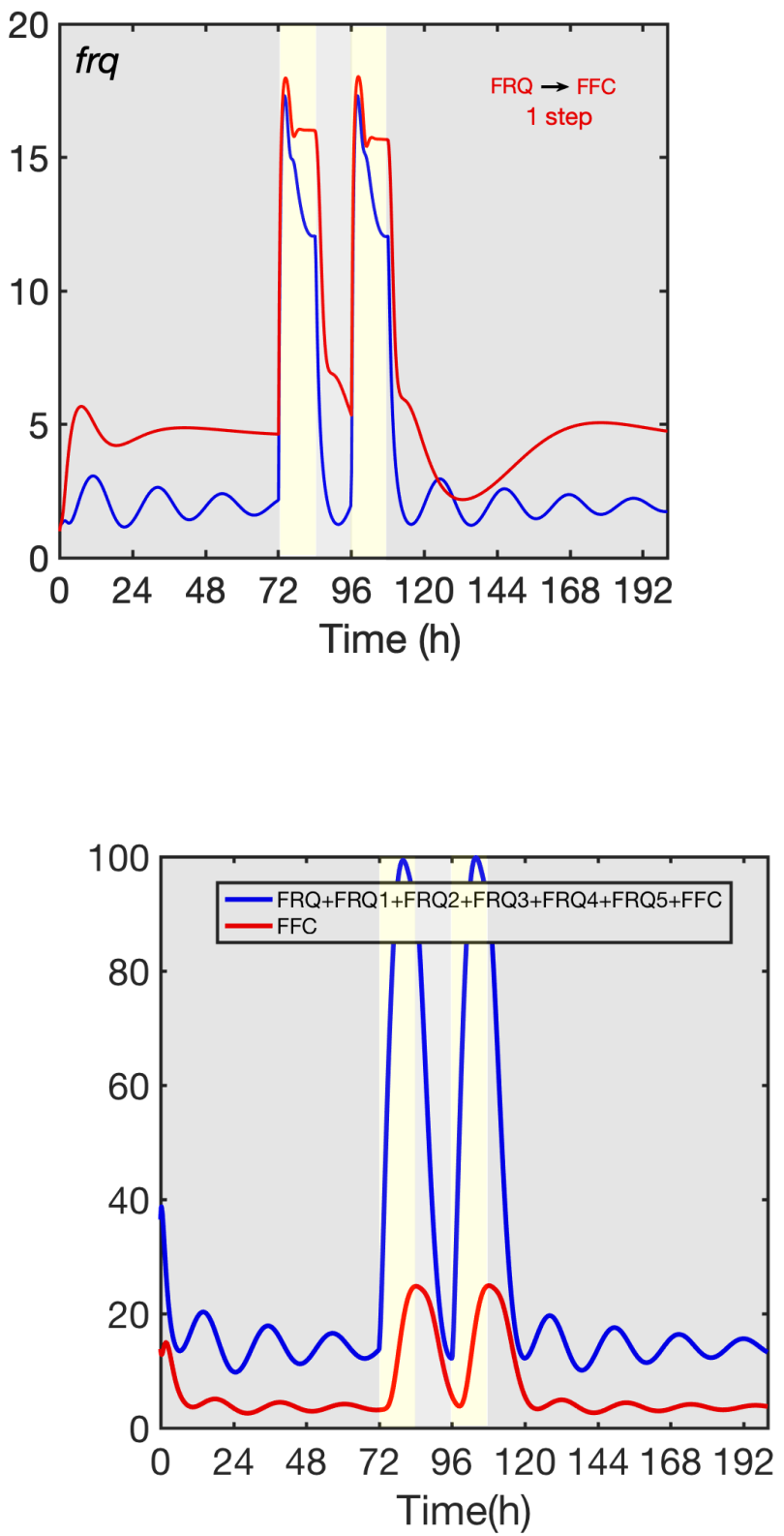
Figure 4. Maturation of inactive FRQ to active FFC is crucial for circadian rhythmicity.

(A) Maturation of FRQ to FFC was modelled by a linear chain of 6 generic steps. The impact of the number of steps on frq expression levels and rhythm is shown. (B) Delay between newly synthesized FRQ and assembled FRH (left panel) and abundance of all unassembled FRQ-species versus assembled FRH (right panel). 
A
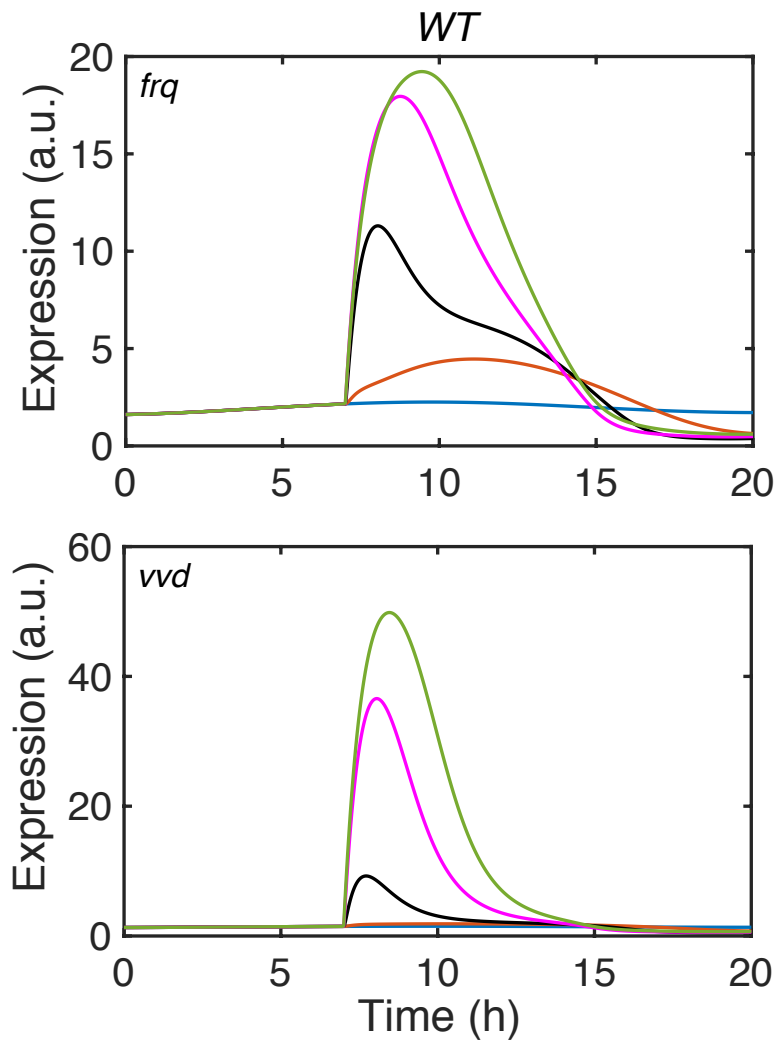

B

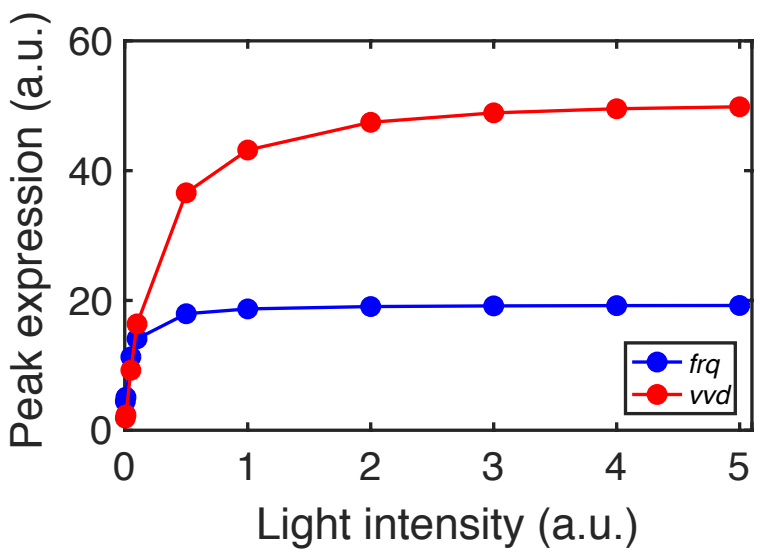

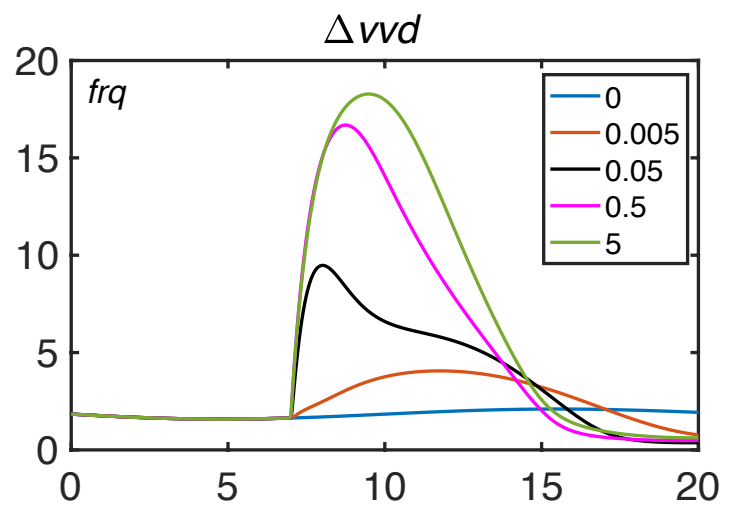
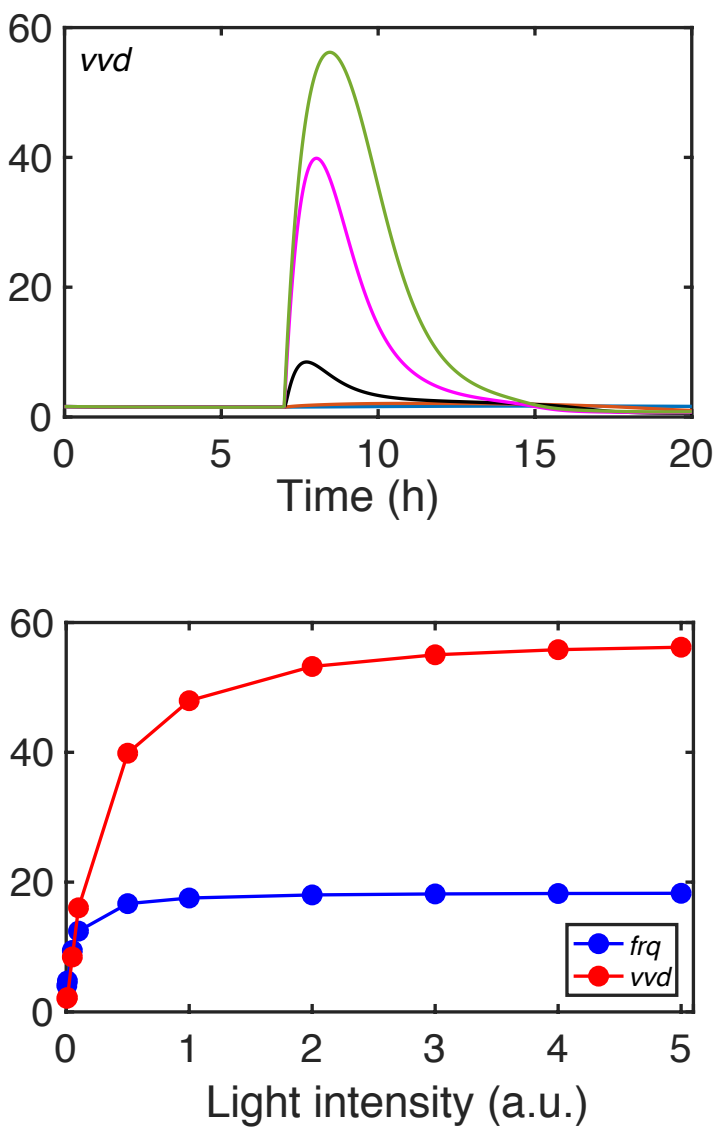
Figure 5. Modelling the response of frq and $v v d$ promoters to light-pulses of different intensity. $W T$ (left panels) and $\Delta v v d$ (right panels) were exposed at $\mathrm{t}=$ 7 min to a virtual LP. (A) Modeled response of frq and $v v d$ to virtual LPs of the indicated intensities. (B) Plot of peak expression levels of frq and $v v d$ versus LP intensity. Simulated LP intensities: $0 ; 0.005 ; 0.005 ; 0.05 ; 0.5$ and 5.0 arbitrary units (a.u.). 

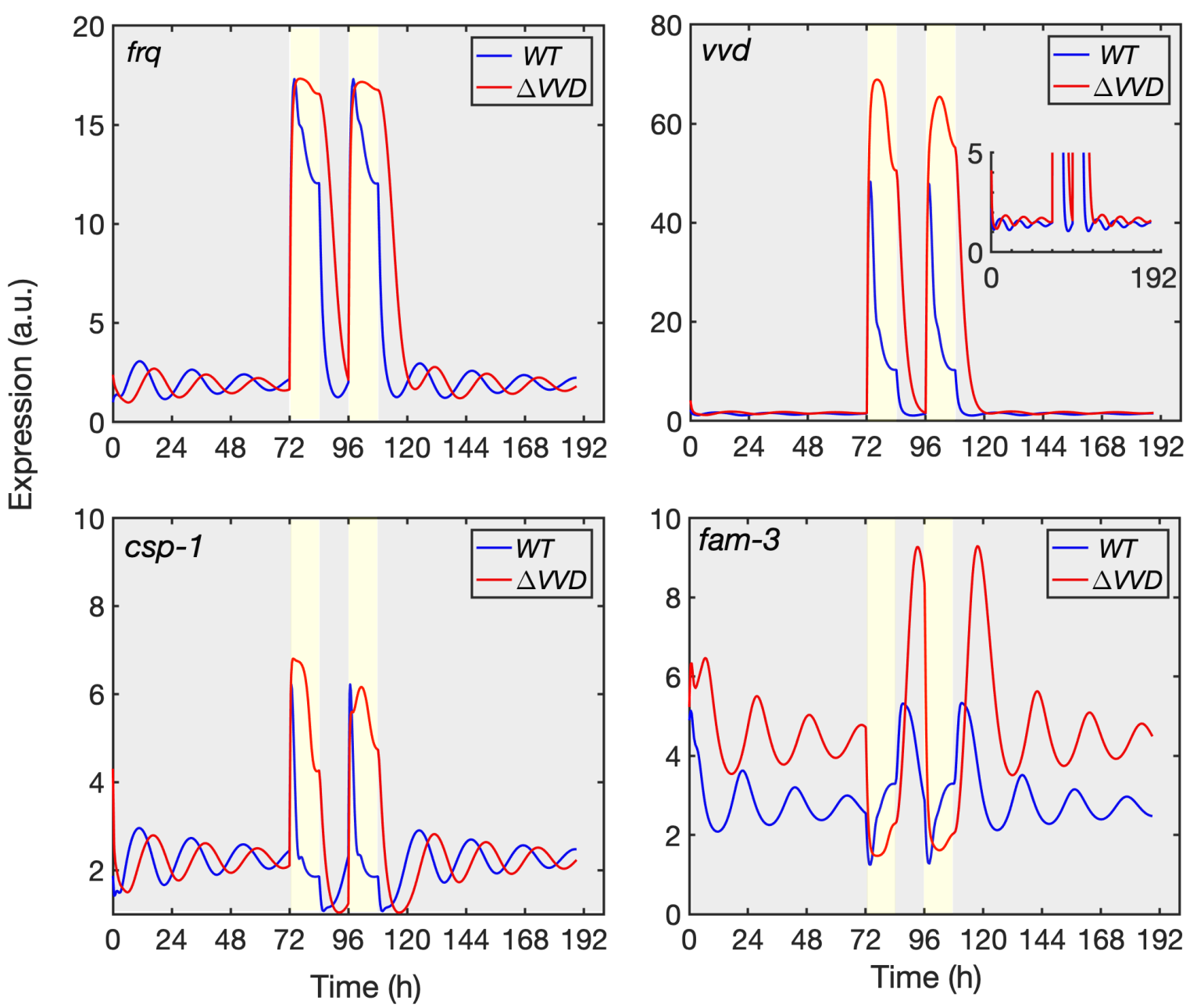
bioRxiv preprint doi: https://doi.org/10.1101/2022.01.24.477555; this version posted February 19,2022 . The copyright holder for this preprint (which was not certified by peer review) is the author/funder, who has granted bioRxiv a license to display the preprint in perpetuity. It is made available under aCC-BY-NC-ND 4.0 International license.

\section{Fig. S1 Phase delay of expression rhythms in $\Delta v v d$}

Modelled trajectories of the expression rhythms in WT and $\Delta v v d$ (see Figure 3) were superimposed. 

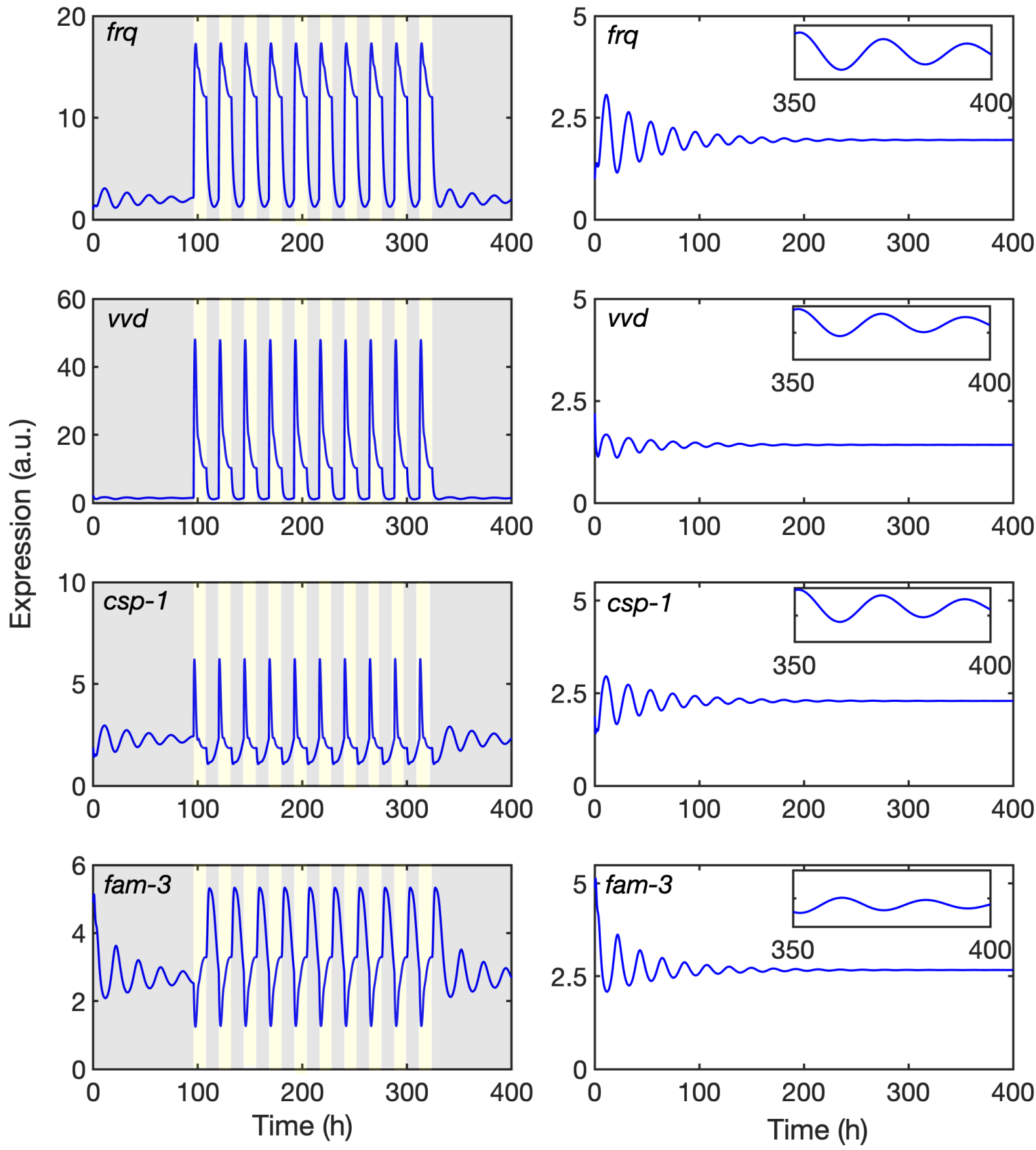
Fig. S2 Characterization of the model.

(A) Simulated response of reporters to 10 repetitive LD cycles. (B) Rhythms of reporter genes dampen in constant darkness. Expression rhythms in the dark of the reporters were modelled for $400 \mathrm{~h}$. Inserts show zoom-in of the expression rhythms between 350 and $400 \mathrm{~h}$. 


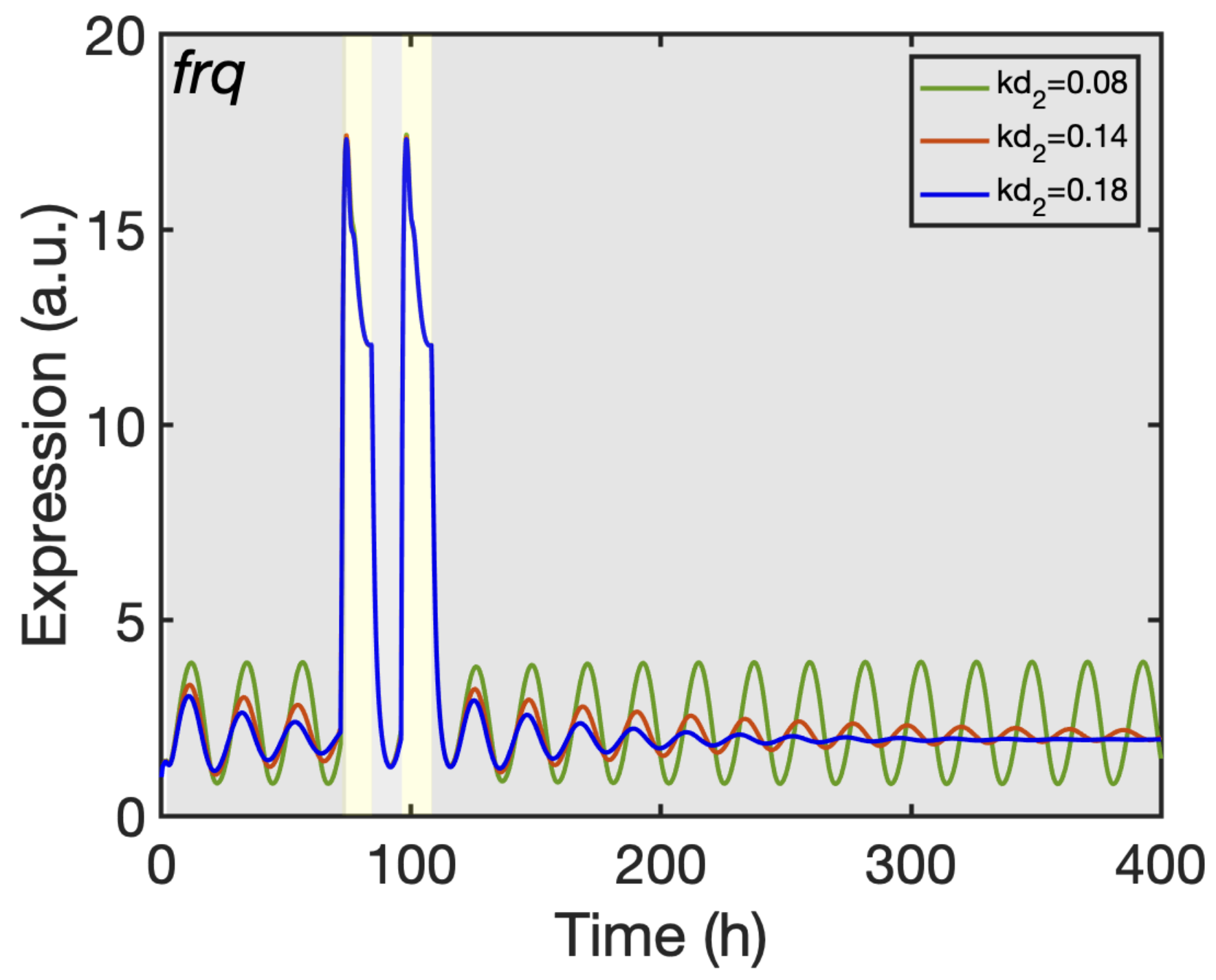


Fig. S3 Impact of WCC dephosphorylation rate on frq expression rhythm The modeled frq expression rhythm damps in the dark (blue trajectory). When the predicted dephosphorylation (reactivation) rate of WCC $\left(\mathrm{k}_{\mathrm{d} 2}=0.18\right)$ was stepwise lowered dampening was reduced $\left(\mathrm{k}_{\mathrm{d} 2}=0.14\right.$, red trajectory) or abolished $\left(\mathrm{k}_{\mathrm{d} 2}=\right.$ 0.08 , red trajectory) and the amplitude increased. 